PII: S0890-6955(97)00055-2

\title{
A SIX-DEGREE-OF-FREEDOM MEASUREMENT SYSTEM FOR THE MOTION ACCURACY OF LINEAR STAGES
}

\author{
K. C. FAN, $\dagger \ddagger$ M. J. CHEN $\S$ and W. M. HUANG $\dagger$
}

(Received 11 July 1997)

\begin{abstract}
This paper presents a new six-degree-of-freedom measurement system (6DMS) which has the capability to measure simultaneously all six motion errors of a linear stage. This system employs three parallel laser beams to detect three relative linear distances of a moving body by Doppler effect so that the positioning, yaw and pitch errors can be separated. With an additional beam splitter and two quadrant photodetectors to detect the lateral shifts of the returned beams, the remaining two straightness errors and roll error can be obtained at the same time. In comparison with the HP5528A system, the accuracy of the positioning error is about $0.01 \mu \mathrm{m}$ to the range of $10 \mathrm{~m}$, the straightness error is about $1 \mu \mathrm{m}$ within the measuring range of $\pm 0.1 \mathrm{~mm}$, and angular errors are all about 1 arcsec within the range of \pm 50 arcsecs. This system is simple in principle and can be easily equipped to any moving stages, such as linear stages, $X-Y$ tables, CMMs, and machine tools. () 1998 Elsevier Science Ltd
\end{abstract}

\section{INTRODUCTION}

Any linear carriage of precision machinery is expected to travel along a straight line and stop precisely at a prescribed position during working. However, in practice, the actual path and orientation of the stage deviate from the straight line of motion due to its own geometric errors. As shown in Fig. 1, a moving table along a specified axis (e.g. the $X$ axis) will inherently have errors in six degrees of freedom, which are three linear errors (linear positioning, horizontal straightness, vertical straightness) and three angular errors (pitch, yaw, roll).

Current technology on the accuracy calibration of linear stages, $X-Y$ tables, CMMs. and machine tools frequently employs the laser interferometer in association with autocollim-

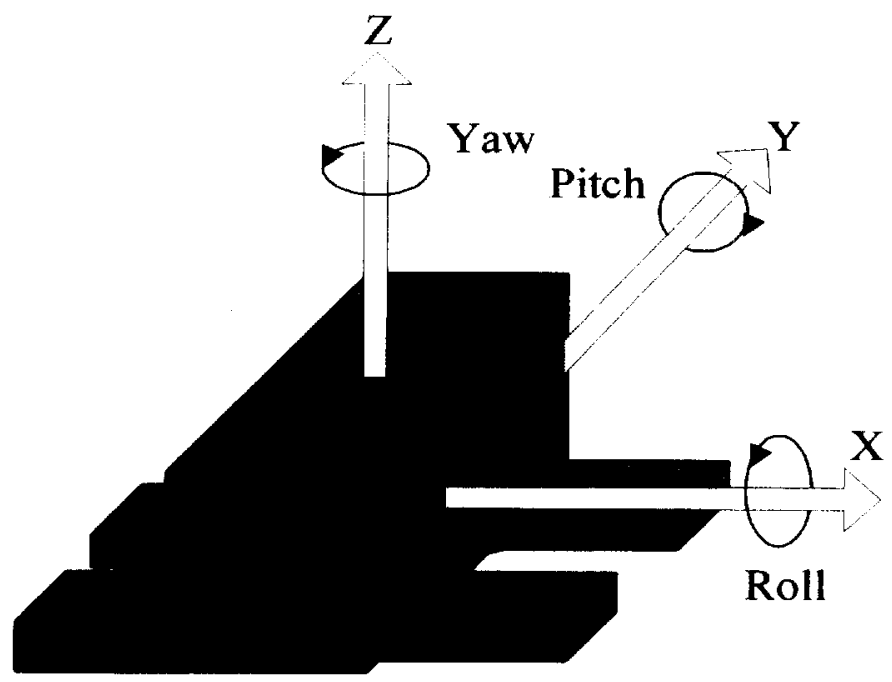

Fig. 1. Linear motion with six degrees of freedom.

†Department of Mechanical Engineering, National Taiwan University, Taipei, Taiwan, R.O.C.

¥Author to whom correspondence should be addressed.

§Department of Mechanical Engineering, Lunghwa Junior College of Technology and Commerce, Taoyuan, Taiwan, R.O.C. 
ator, electronic level, mechanical references, and LVDTs for the six-degree-of-freedom errors of each axis [1]. This process often takes a long time to finish the entire job as each error should be calibrated separately. Besides, the set-up of the measuring devices and the alignment of the reference axis are tedious. Normally, as time elapses the environmental temperature changes accordingly. Most working environments do not provide a temperature control facility. It is obvious that the measurement uncertainty could increase as the process time increases. A breakthrough in the technology to have a multi-function measuring device and to reduce the measuring time is urgently needed.

Although there is no such kind of commercial product available in the market so far, there are some research reports of multi-error detectors published in recent years. Almanar [2] and Fang and Fan [3] both developed a multi-function error calibration system for NC machine tools by using three sets of quadrant photodetectors to measure simultaneously five-degree-of-freedom errors. The MDFM system developed by Ni et al. $[4,5]$ is regarded as the most advanced system for CMM geometric errors. His system employs four quadrant detectors for five-degree-of-freedom measurement, except the positioning error. Shimizu et al. [6] also proposed a simultaneous measuring method for table motion errors in six degrees of freedom by using three quadrant photodetectors and a single-axis interferometric system. Other systems which detect more than one degree of error can be done by the taut-wire system [1,7], or the laser Doppler displacement meter [8, 9]. In this paper, a complete solution is proposed by the use of a six-degree-of-freedom measurement system (6DMS) which employs the interferometric principle for pitch and yaw, rather than the beam position signals as chosen by the above-mentioned methods. The whole 6DMS is very simple in principle, very compact in structure, and very accurate in performance.

\section{PRINCIPLE OF MEASUREMENT}

\subsection{System configuration}

Figure 2 shows the schematic diagram of the 6DMS. It includes a moving part which is fixed onto the moving stage and a stationary part which can be mounted on a reference table. The moving part consists of three retroreflectors. The stationary part consists mainly of a laser head unit with three parallel laser sources, a beam splitter. and two quadrant detectors. The laser head unit employs a dual-beam laser Doppler displacement meter (LDDM, model 2002 made by Optodyne Co.), and a single-beam LDDM (model 1000) as the light sources, which are parallel to each other. In order to minimize the cosine errors among the three displacement measurements and to ensure angular accuracy for pitch and yaw measurements, the parallelism of these three beams is precisely adjusted in advance. Each beam emitting from the laser head unit is reflected by the corresponding retroreflector mounted on the moving stage. Therefore, the reflectors can reflect these laser

1. Quadrant photo detector *2

2. Retroreflector * 3

3. Beam splitter

4. LDDM 2002 (Dual laser beam)

5. LDDM 1000 (Single laser beam)

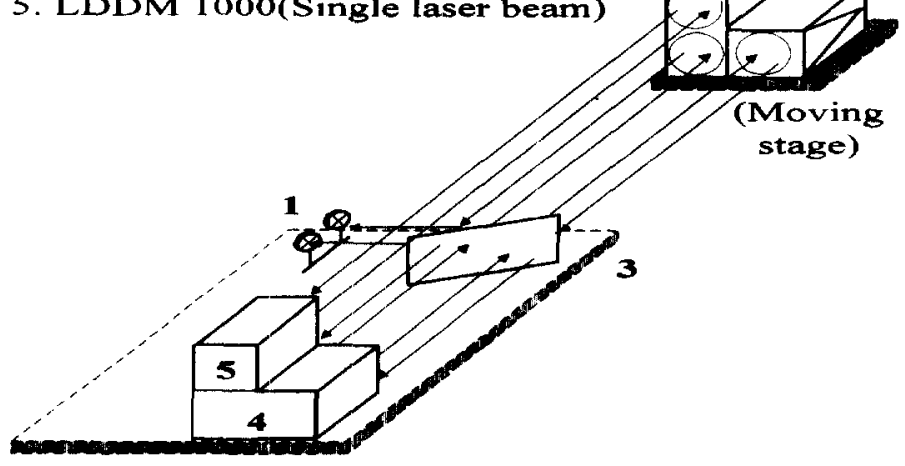

(Reference table)

Fig. 2. Schematic diagram of the 6DMS. 
beams back to the receivers of the laser head unit. Comparing the relative linear displacements of these three retroreflectors, the positioning, pitch and yaw errors could be discovered. Two of the reflected beams from retroreflectors are split by a large $50 / 50$ beam splitter before entering into the laser head unit. Each split beam is received by a corresponding quadrant photodetector [9]. Comparing the horizontal and vertical deviations of laser spots on the two quadrant detectors, the errors of two directional straightness and the roll error of the moving stage can be obtained.

\subsection{Measurement of displacement, pitch and yaw errors}

The LDDM is based on the principles of radar, the Doppler effect and optical heterodyne [7]. Basically, a target or retroreflector is illuminated by a laser beam. The laser beam reflected by the retroreflector is frequency shifted by the motion of the retroreflector, and the phase of the reflected beam is proportional to the position of the retroreflector. The displacement of motion is

$$
X=\frac{c}{2 f}\left(N+\frac{\phi}{2 \pi}\right)
$$

where $c$ is the speed of light, $f$ is the laser frequency, $N$ is the number of $2 \pi \mathrm{s}$, and $\phi$ is the phase angle.

In the designed system, by making three simultaneous distance measurements $\left(L_{1}, L_{2}\right.$, $L_{3}$ ) parallel to the motion axis, one can obtain displacement, pitch error and yaw error of the moving body at a time. The actual displacement $(L)$ of the moving body can be obtained from the dual-beam LDDM as

$$
\text { Displacement }(L)=\left(L_{1}+L_{2}\right) / 2 \text {. }
$$

The pitch error $\left(\theta_{v}\right)$ can be computed by the difference between two laser displacements in the vertical plane divided by the separation of these two beams $\left(D_{v}\right)$ :

$$
\text { Pitch error }\left(\theta_{y}\right)=\left(L_{1}-L_{3}\right) / D_{\mathrm{v}} \text { rad }
$$

where $D_{v}$ is equal to $86.147 \mathrm{~mm}$ in the 6DMS. Similarly, the yaw error $\left(\theta_{*}\right)$ can be obtained by the difference between two laser displacements in the horizontal plane divided by the separation of these two beams $\left(D_{\mathrm{h}}\right)$ :

$$
\text { Yaw error }\left(\theta_{3}\right)=\left(L_{2}-L_{1}\right) / D_{\mathrm{h}} \text { rad }
$$

where $D_{\mathrm{h}}$ is equal to $27.940 \mathrm{~mm}$ in the 6DMS.

\subsection{Measurement of straightness errors}

The quadrant photodetector (QD), which contains four quadrants of photodiodes, can be used to determine the position of the spot centroid deviated from its center [1, 10]. As shown in Fig. 3, when a stage is moved along the axis of travel, with a certain lateral

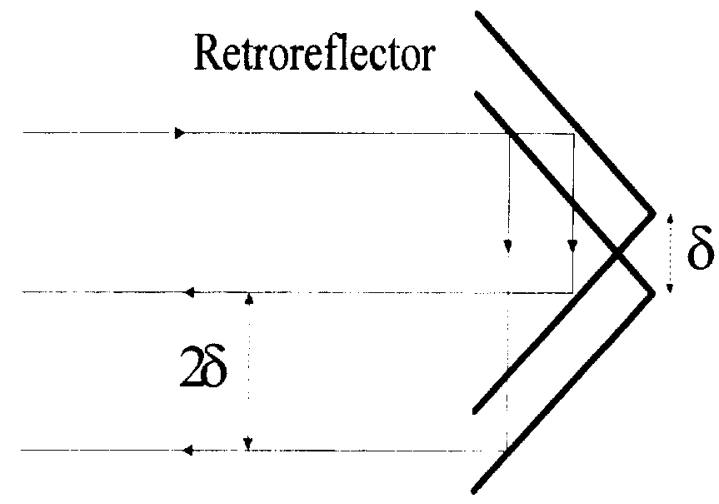

Fig. 3. Measuring principle of straightness error. 
distance $(\delta)$ the laser beam reflected from the retroreflector and split by the beam splitter will be shifted on the corresponding QD by a distance of $2 \delta$. Thus, the sensitivity of the straightness error measurement can be improved by a factor of 2 .

Applying the above principle, the straightness errors of the moving stage can be obtained from the horizontal and vertical positions of laser spots on $\mathrm{QD}_{1}$ and $\mathrm{QD}_{2}$ as:

$$
\begin{aligned}
& \text { Horizontal error: } \Delta Y=\left(h_{1}+h_{2}\right) / 4 \\
& \text { Vertical error: } \Delta X=\left(v_{1}+v_{2}\right) / 4
\end{aligned}
$$

where $\left(h_{1}, v_{1}\right)$ and $\left(h_{2}, v_{2}\right)$ are the outputs of $\mathrm{QD}_{1}, \mathrm{QD}_{2}$ respectively.

\subsection{Measurement of roll error}

As the stage (or the retroreflector unit) is moved along the axis of travel with a rotation about this axis, each reflected beam from the retroreflector will change its vertical position on the corresponding QD. The roll error can be calculated from the relative vertical deviations of the two QDs as:

$$
\text { Roll error }\left(\theta_{x}\right)=\left(v_{2}-v_{1}\right) /(2 S)
$$

where $S$ denotes the distance between the two lower retroreflectors and is identical to $D_{\mathrm{h}}$.

\section{SYSTEM CALIBRATION}

Calibration tests for the six-degree-of-freedom errors of the 6DMS were carried out with a HP 5528A interferometer measurement system as the reference standard.

\subsection{Calibration of straightness errors}

Fig. 4(a) and (b) show the experimental set-up for the calibration tests of straightness errors on an $X-Y$ stage. The $X-Y$ stage is moved within a range from -0.5 to $+0.5 \mathrm{~mm}$, at an approximate increment of $50 \mu \mathrm{m}$ along each axis respectively. Figure 5(a) and (b) show the horizontal and vertical characteristic curves. The accuracy of straightness measurement of the 6DMS is found about $\pm 5 \mu \mathrm{m}$ within the measuring range of $\pm 0.5 \mathrm{~mm}$. However, within the range of $\pm 0.1 \mathrm{~mm}$ the accuracy can be maintained to within $\pm 1 \mu \mathrm{m}$ and this is the error range most linear stages can reach.

\subsection{Calibration tests of angular errors}

Figure 6(a)-(c) shows the experimental set-up for the performance tests of roll, pitch and yaw errors, respectively. The tests of the roll and pitch error were carried out with two adjustable jacks. By adjusting the height of each jack, the roll and pitch motions could

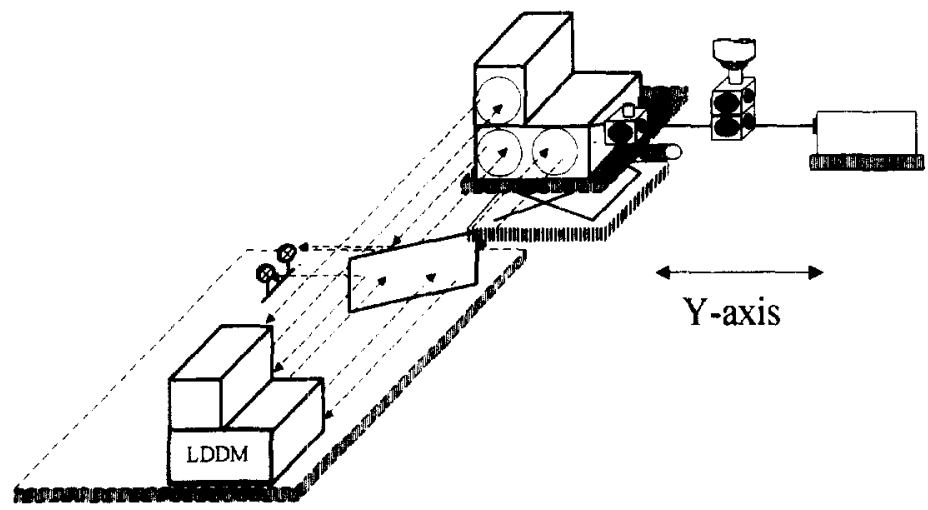

(a)

Fig. 4. Set-up for straightness calibration: (a) horizontal straightness; (b) vertical straightness. 


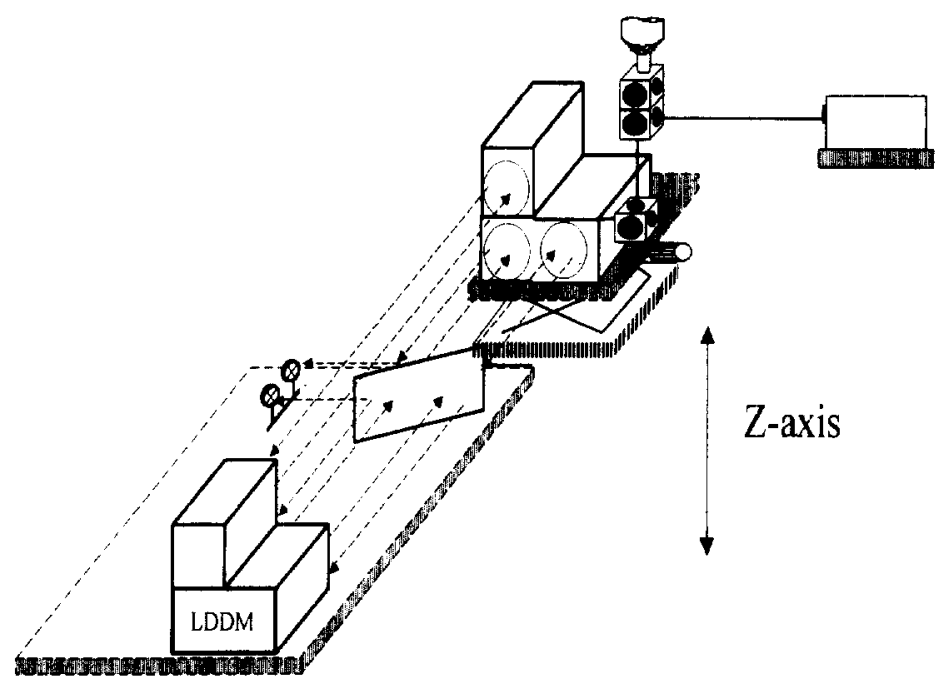

(b)

Fig. 4. Continued.

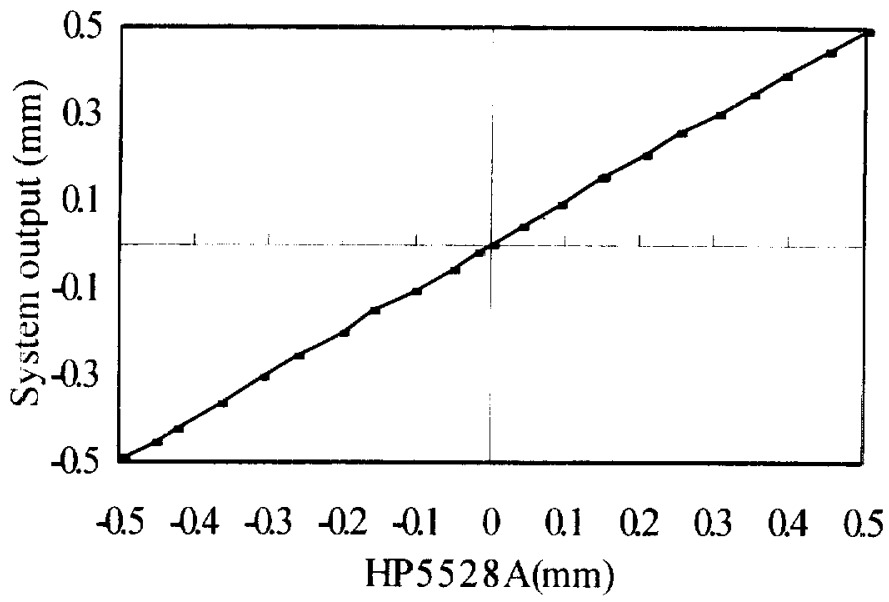

(a)

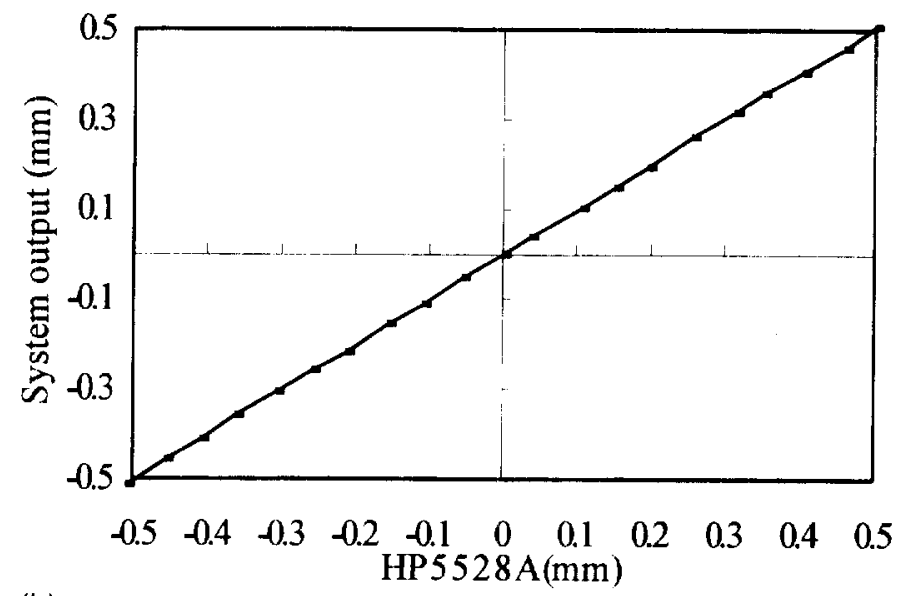

(b)

Fig. 5. Results of calibration: (a) horizontal straightness; (b) vertical straightness. 


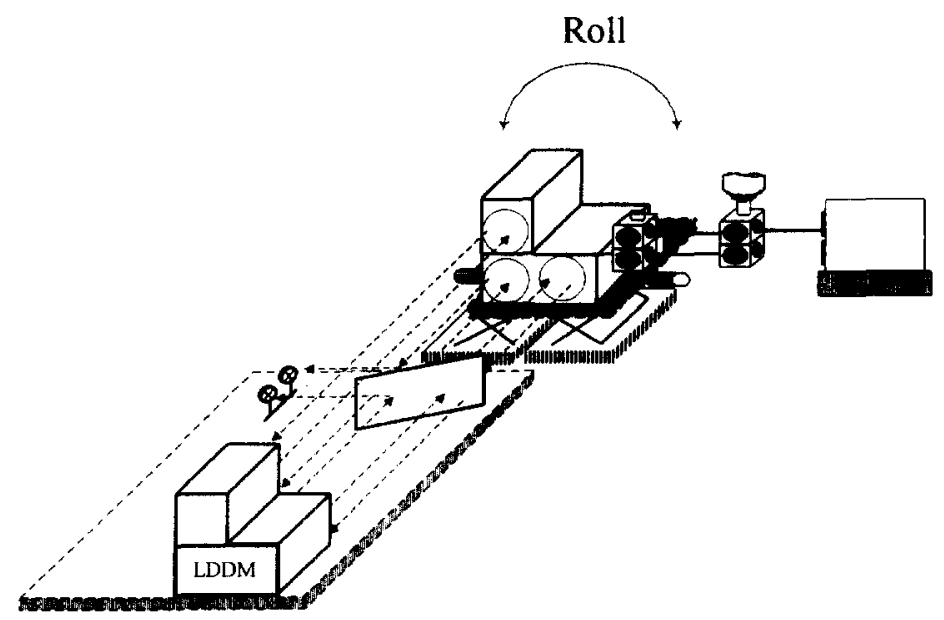

(a)

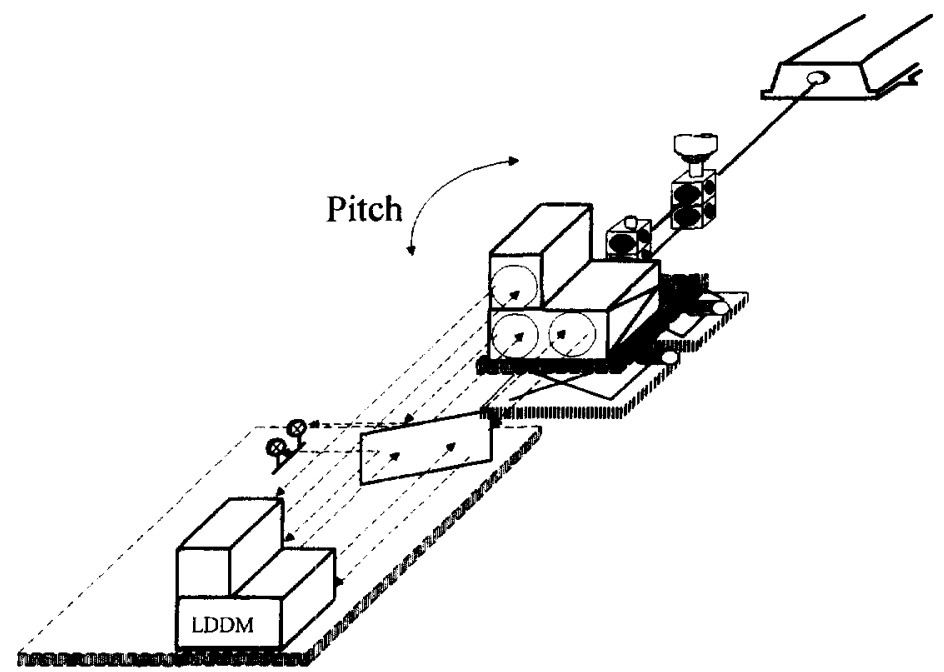

(b)

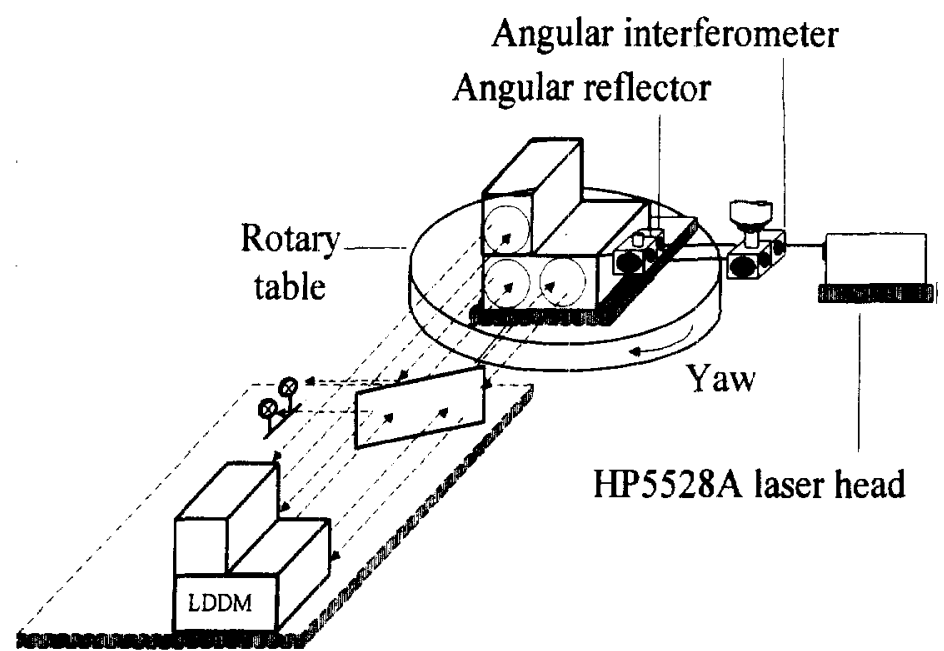

(c)

Fig. 6. Set-up for angular calibration: (a) roll; (b) pitch; and (c) yaw. 
be created. For the yaw error, calibration was performed by rotating the rotary table at an increment of approximate 2 arcsecs. The ranges of tests are from -500 to +500 arcsecs for the roll error, and from -50 to +50 arcsecs for the pitch and yaw errors. Figure 7 (a)-(c) shows the results of the roll, pitch and yaw errors, respectively. Comparing the 6DMS with the HP system, the calibrated roll, pitch and yaw errors are all about 1 arcsec within the measuring range of \pm 50 arcsecs.

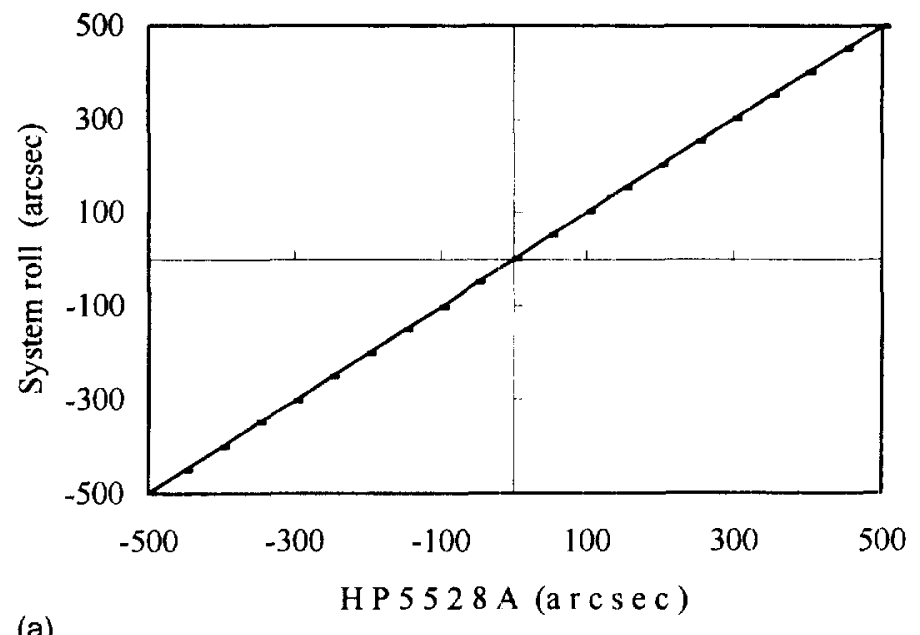

(a)

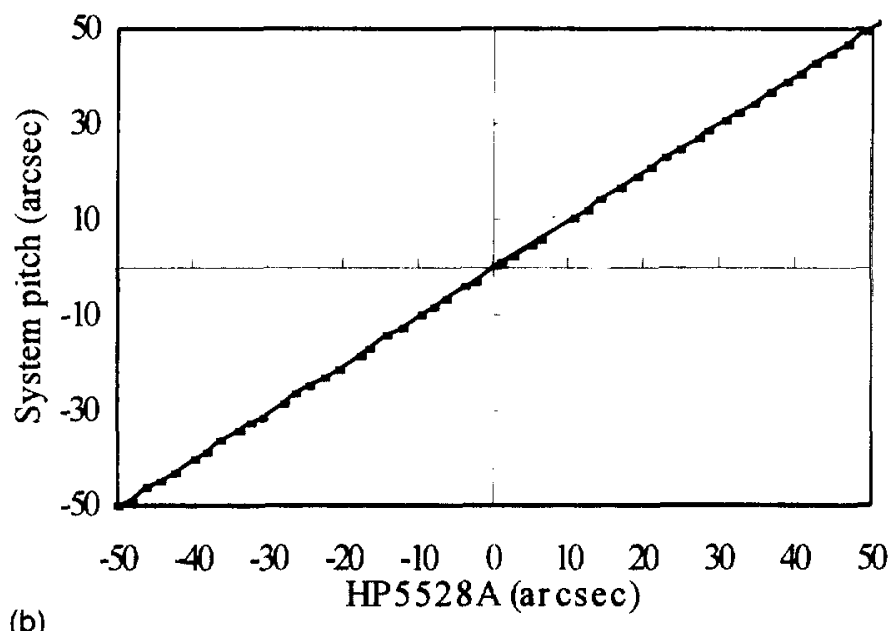

(b)

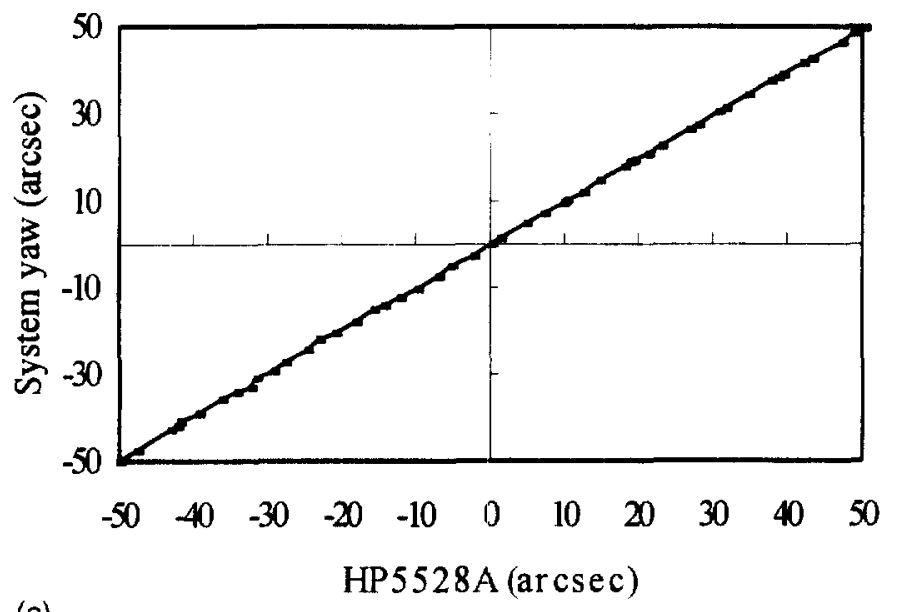

(c)

Fig. 7. Results of angular calibration: (a) roll; (b) pitch; and (c) yaw. 


\section{LXPERIMENTAL TESTS}

Figure 8(a) and (b) shows the system diagram and its photo of the experimental set-up to test the motion error of a linear stage. All the elements are secured on an optical table to avoid vibration from the floor. The system may measure all six degrees of error simultaneously on a specified position. Measurements were performed by moving the linear stage for a travel of $200 \mathrm{~mm}$ with an increment of $10 \mathrm{~mm}$, for a number of three runs. Figures 9-13 show the motion errors of the stage. In the experiment, since the roll error of the linear stage is very small. the system may not effectively respond its changes. However, through improving the resolution of the QDs or increasing the distance between the two lower laser heads, it is expected that the performance of the developed system will be improved effectively.

\section{CONCLUSION}

This paper represents a new system to measure simultaneously the six-degree-of-freedom errors along an axis of motion. It is suitable for the calibration of the linear stage, CMM, machine tool, precision $X-Y$ stage and other linear moving devices. In addition, the moving part is wireless which eliminates any induced pushing and pulling errors of

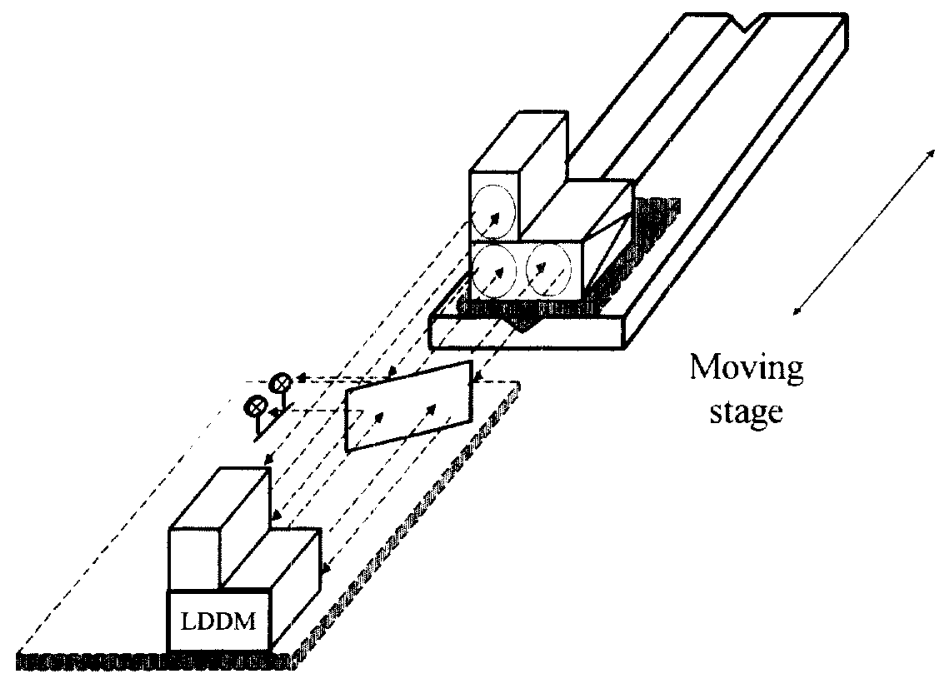

(a)

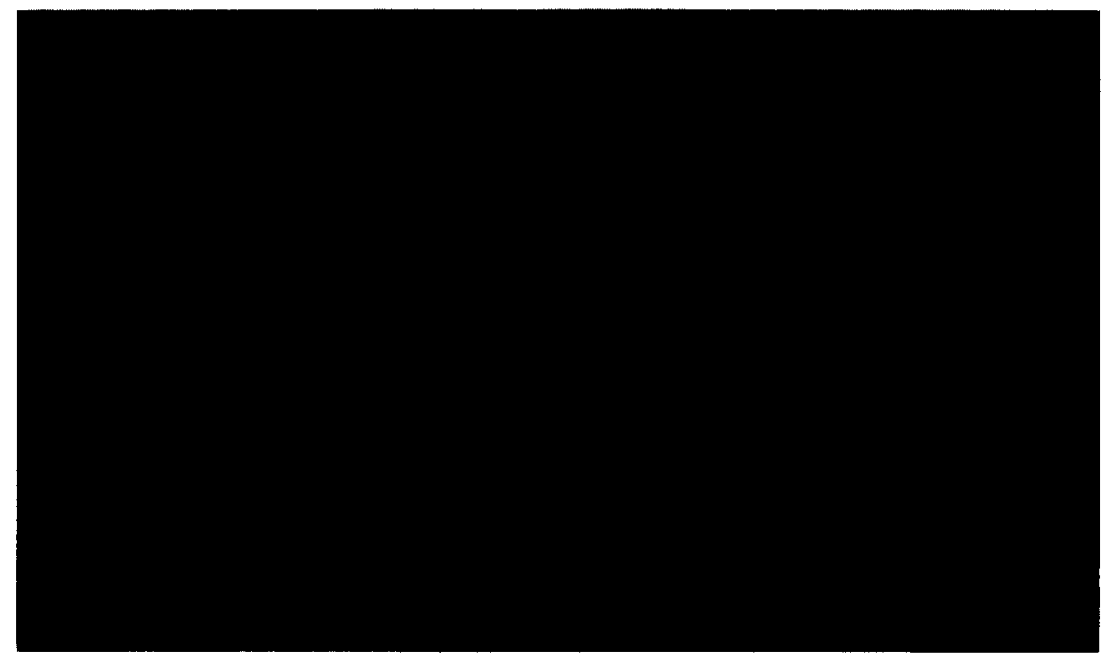

(b)

Fig. 8. Accuracy test of linear slage: (a) experimental set-up: (b) photo of the experimental set-up. 


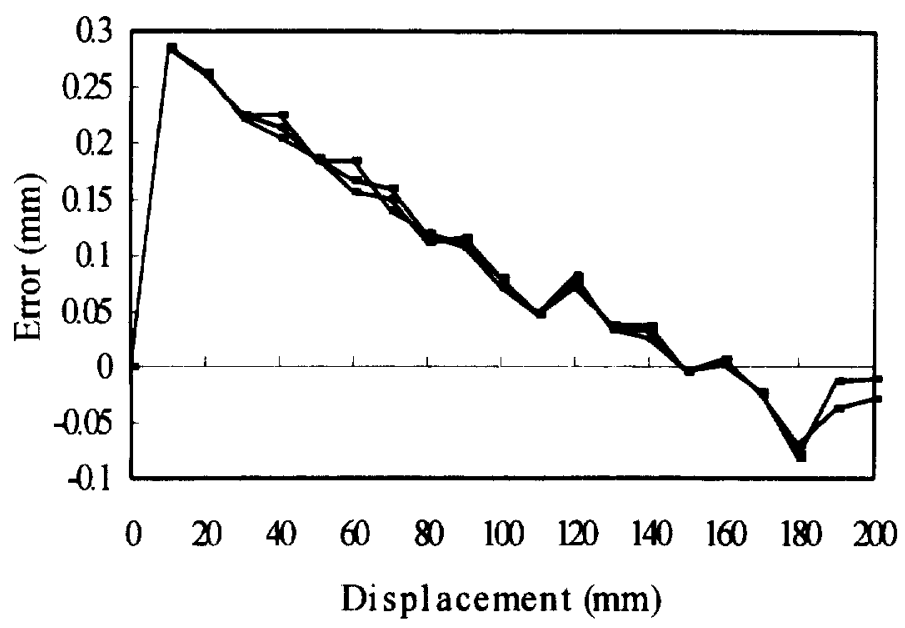

Fig. 9. Positioning error of the stage.

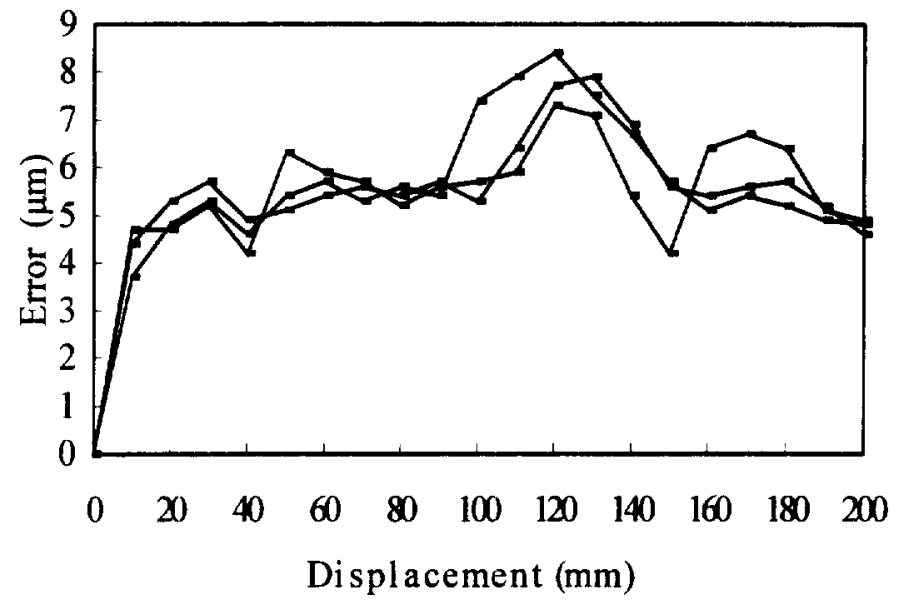

Fig. 10. Horizontal straightness error of the stage

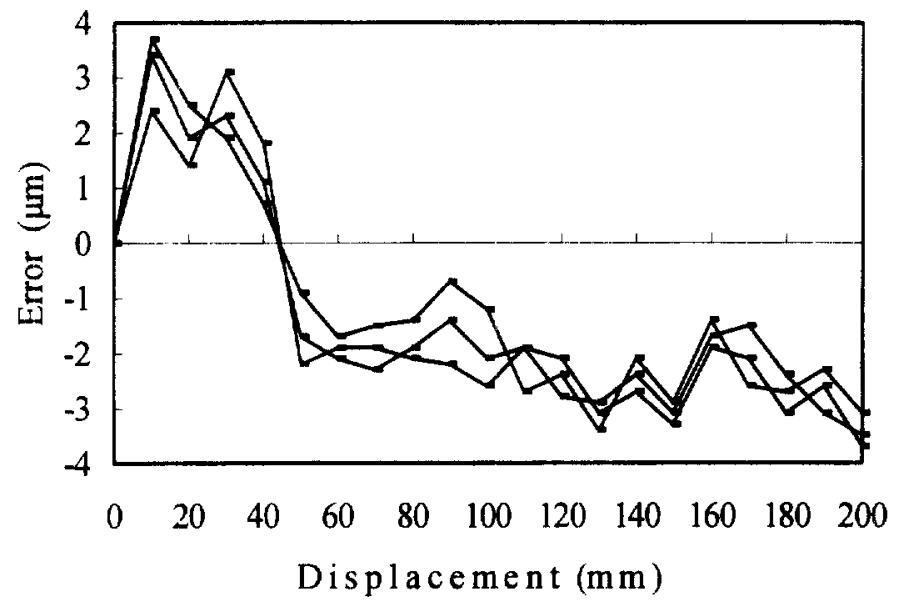

Fig. 11. Vertical straightness error of the stage.

the wire. The developed system is simpler in principle and lower in cost in comparison with the HP laser interferometer system or other developed systems. 


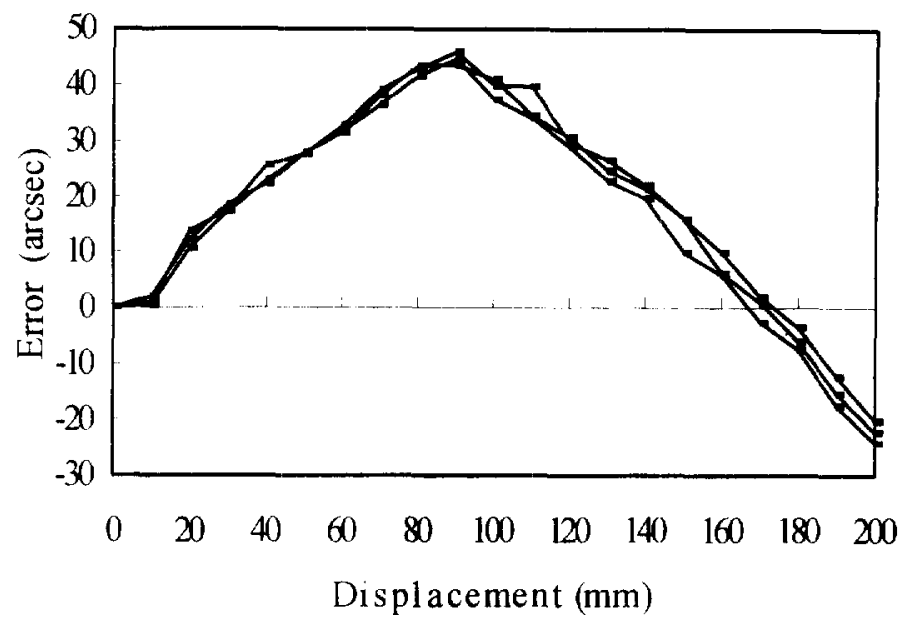

Fig. 12. Pitch error of the stage.

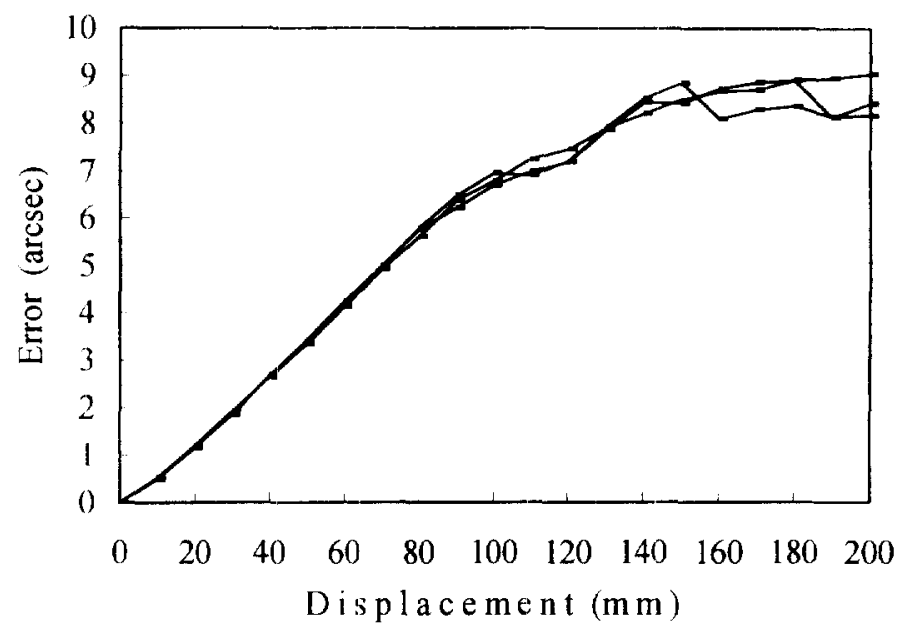

Fig. 13. Yaw error of the stage.

Acknowledgement-This report forms part of the project funded by the National Science Council of ROC on the Development of High Precision Stages.

\section{REFERENCES}

[1] Weck, M. M., Geometric and kinematic errors. Technology of Machine Tools, 1980. 5, 9-12.

12] Almanar, I. P. A study of pre-calibrated techniques for compensation of geometrical errors on large machine tools. Ph.D. Thesis, UMIST, 1986.

[3] Fang, C. Y. and Fan, K. C., Development of multi-function error calibration system for NC machine tools. In Proc: 3rd ROC-ROK Metrology Symposium, Taipei. Taiwan, 1990, pp. 163-171.

[4] Ni. J., Huang, P. S. and Wu, S. M.. A multi-degree-of-freedom measuring system for CMM geometric errors. J. Engng Ind., Trams. ASME, 1992, 114, 362-369.

[5] Huang, P. S. and Ni, J., On-line error compensation of coordinate measuring machines. Int. J. Mach. Tools Manufact., 1995, 35, 725-738.

[6] Shimizu, S., Lee, H. -S. and Imai, N., Simultaneous measuring method of table motion errors in 6 degrees of freedom. Int. I. Japan Soc: Precivion Engng, 1994, 28, 273-274.

[7] Chang, K. Y.. Design and application of a laser-based taut-wire sensor system for machine tools alignment testing. M.Sc. Thesis, UMIST. 1994.

18] Wang, C. P.. Lalser Doppler displacement measurement. Lasers \& Optronics, 1987, 9, 69-71.

[9] Wang, G. P., Ulsing the laser Doppler displacement meter for precision positioning and motion control. Motion Control, June, 1991.

[10] Madden. R. M., Silicon position sensing detectors for precision measurement and control. Adv. Opt. Metrol., Proce SPIE, 1978, 153,101-107. 